\title{
Cerebral Perfusion Changes in Post-Concussion Syndrome: A Prospective Controlled Cohort Study
}

\author{
Karen M. Barlow, ${ }^{1-3}$ Lorenzo D. Marcil, ${ }^{4}$ Deborah Dewey, ${ }^{1,3,5}$ Helen L. Carlson,,6 \\ Frank P. MacMaster, ${ }^{1,3,7-9}$ Brian L. Brooks, ${ }^{1-3,6,13}$ and R. Marc Lebel ${ }^{3,10-12}$
}

\begin{abstract}
The biology of post-concussive symptoms is unclear. Symptoms are often increased during activities, and have been linked to decreased cerebrovascular reactivity and perfusion. The aim of this study was to examine cerebral blood flow $(\mathrm{CBF})$ in children with different clinical recovery patterns following mild traumatic brain injury (mTBI). This was a prospective controlled cohort study of children with mTBI (ages 8 to 18 years) who were symptomatic with postconcussive symptoms at one month post-injury (symptomatic, $n=27$ ) and children who had recovered quickly (asymptomatic, $n=24$ ). Pseudo continuous arterial spin labeling magnetic resonance imaging (MRI) was used to quantify CBF. The mTBI groups were imaged at 40 days post-injury. Global and regional CBF were compared with healthy controls of similar age and sex but without a history of mTBI $(n=21)$. Seventy-two participants (mean age: 14.1 years) underwent neuroimaging. Significant differences in CBF were found: global CBF was higher in the symptomatic group and lower in the asymptomatic group compared with controls, $(\mathrm{F}(2,69) 9.734 ; p<0.001)$. Post-injury symptom score could be predicted by pre-injury symptoms and CBF in presence of mTBI (adjusted $\mathrm{R}^{2}=0.424 ; p<0.001$ ). Altered patterns of cerebral perfusion are seen following mTBI and are associated with the recovery trajectory. Symptomatic children have higher CBF. Children who "recovered" quickly, have decreased CBF suggesting that clinical recovery precedes the cerebral recovery. Further longitudinal studies are required to determine if these perfusion patterns continue to change over time.
\end{abstract}

Keywords: arterial spin labeling; cerebral blood flow; pediatric brain injury; recovery; traumatic brain injury

\section{Introduction}

$\mathbf{T}$ HE POTENTIAL FOR LONG-TERM CONSEQUENCES after mild traumatic brain injury (mTBI) (including concussion) is a significant public health concern. As many as one in five children will sustain an mTBI before the age of 16 years, ${ }^{1,2}$ and $13 \%$ continue to have post-concussive symptoms for 3 months or longer., Although the pathophysiology of the acute injury is fairly well understood, the neurobiological explanations for prolonged symptoms are not. ${ }^{5,6}$ As we search for better treatments, it becomes very important to try to determine the neuropathological underpinnings of poor outcome.

Routine clinical neuroimaging has not been able to provide a biological marker for persistent post-concussive symptoms. These symptoms are often increased during activities such as homework and physical exercise, ${ }^{7,8}$ and have been linked to increased cortical network activation ${ }^{9,10}$ and decreased cerebrovascular reactivity. ${ }^{11,12}$ Alterations in cerebral blood flow (CBF), although well documented in moderate/severe TBI, is not well described in mTBI and post-concussion syndrome (PCS) ${ }^{13-15}$ For example, Maugans and colleagues reported an overall decrease in $\mathrm{CBF}$ in 11 children at a variety of time-points following mTBI, and although most children had a good recovery there was considerable CBF heterogeneity within the sample. ${ }^{16}$ It is important to examine the contribution of $\mathrm{CBF}$ alterations as decreased cerebral perfusion in a condition of increased metabolic requirement (i.e., with increased network activation or exercise) could lead to an increase in secondary injury and/or an exacerbation of symptoms of PCS. In addition, cerebral perfusion could be an objective biomarker of cerebral recovery in children with mTBI.

\footnotetext{
${ }^{1}$ Department of Pediatrics, ${ }^{2}$ Department of Clinical Neurosciences, ${ }^{4}$ Health Sciences, ${ }^{5}$ Department of Community Health Sciences, ${ }^{9}$ Department of Psychiatry, ${ }^{10}$ Department of Radiology, ${ }^{11}$ Biomedical Engineering, ${ }^{13}$ Department of Psychology, University of Calgary, Calgary, Alberta, Canada.

${ }^{3}$ Alberta Children's Hospital Research Institute, Calgary, Alberta, Canada.

${ }^{6}$ Alberta Children's Hospital, Calgary, Alberta, Canada.

${ }^{7}$ Strategic Clinical Network for Addictions and Mental Health, Alberta Health Services, Edmonton, Alberta, Canada.

${ }^{8}$ Mathison Centre for Mental Health Research and Education, Hotchkiss Brain Institute, Calgary, Alberta, Canada.

${ }^{12}$ GE Healthcare, Calgary, Alberta, Canada.

(c) Karen M. Barlow et al., 2016; Published by Mary Ann Liebert, Inc. This Open Access article is distributed under the terms of the Creative Commons Attribution Noncommercial License (http://creativecommons.org/licenses/by-nc/4.0/) which permits any noncommercial use, distribution, and reproduction in any medium, provided the original author(s) and the source are credited.
} 
Arterial spin labeling (ASL) is a quantitative method of assessing cerebral tissue perfusion using magnetic resonance imaging (MRI). ${ }^{17}$ It is performed without manipulating the cerebral circulation (e.g., using inhaled gases) or contrast agents and is therefore particularly suitable for research with children. ${ }^{18}$ It is intrinsically quantitative and reproducible allowing it to be used for longitudinal evaluation. ${ }^{19}$ The aim of this study was to examine cerebral perfusion changes using ASL-MRI in children with different clinical recovery patterns following mTBI. Specifically, we investigated whether children who had failed to recover at one-month postinjury (symptomatic) had different CBF compared with children who had recovered (asymptomatic) and healthy control children. We hypothesized that children with ongoing symptoms would have different cerebral perfusion when compared with children who had recovered following an mTBI and with healthy controls.

\section{Methods}

This prospective controlled cohort study was performed as part of the Play Game Trial, which is a randomized controlled trial of melatonin for the treatment of PCS following mTBI (https:// clinicaltrials.gov/ct2/show/NCT01874847). ${ }^{20}$ This is a randomized controlled treatment trial of post-concussion symptoms in children using melatonin for 4 weeks and involved comprehensive assessments before and after treatment including imaging and cognitive assessments. Here, we report pre-treatment assessments. Ethical clearance was granted by the University of Calgary Conjoint Health Research Ethics Board (REB13-0372).

\section{Participants}

Potential participants were identified from children with an mTBI presenting to the emergency room (ER) or the concussion clinic at the Alberta Children's Hospital (ACH) within 2 weeks of injury. mTBI was defined as an impact to the head or body resulting in at least one of the following: an observed loss of consciousness $(<30 \mathrm{~min})$, a Glasgow Coma Score of 13-15, or at least one acute symptom suggesting neurological dysfunction attributable to the injury (e.g., headache, confusion, vomiting, amnesia, balance problems). ${ }^{3,21,22}$ Concussion is considered to be part of the spectrum of mTBI. ${ }^{23}$ Consent to contact was obtained in 231 potential participants at 3 to 4 weeks post-injury (see Fig. 1). Clinical and neuroimaging assessments were performed at approximately 40 days post-injury.

Inclusion criteria. Included children (8 to 18 years) with mTBI who consented to telephone follow-up at 4 weeks post-injury.

Exclusion criteria. Included suspected child abuse, alcohol or drug use at the time of injury, significant past medical/psychiatric history, English as a second language, a previous TBI within the last 3 months, failure to recover from a previous mTBI, contraindications/inability to have an MRI scan, and/or the use of psychoactive medications.

Children with a history of mild learning difficulties or attentional problems were not excluded. One-hundred sixteen children were contacted. Consent was obtained in 53 participants. Healthy controls satisfying the exclusion criteria and without a history of TBI were recruited $(n=21)$. These children were identified by mTBI

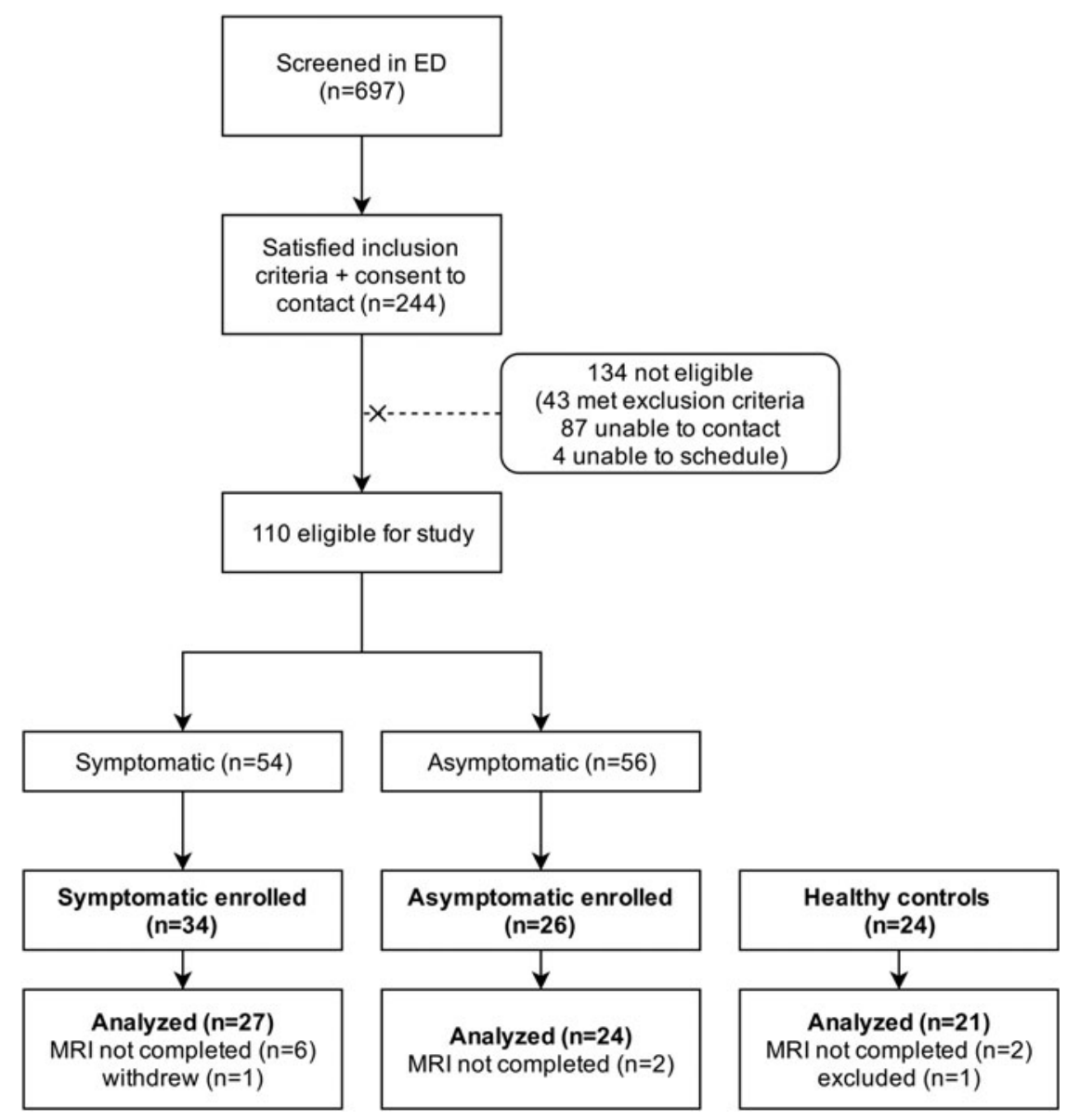

FIG. 1. Consort diagram depicting enrollment through to analysis: Children with mTBI were identified in the ED ( $n=244)$ and enrolled at 3 to 4 weeks post-injury $(n=84)$. ED, emergency department; mTBI, mild traumatic brain injury. 
participants, that is, siblings or friends. As perfusion is known to vary during childhood and adolescence, healthy controls were selected to be similar in age and sex. ${ }^{24}$

Post-Concussion Symptom Inventory (PCSI). This is an age-appropriate, standardized questionnaire that was used to assess PCS symptoms. This validated questionnaire provides ratings for 26 symptoms and an overall rating of PCS symptoms following mTBI. ${ }^{4,25-27}$ Individual scores for each symptom (0-6), a total symptom score (0-156), and a "degree of difference from preinjury" score (0-4) were obtained. The participants completed the questionnaire for their pre-injury status at enrollment, and their post-injury PCSI score was performed on the day of the MRI scan.

Recovery status. Children were considered to be symptomatic if they reported a 2-point increase in two or more symptoms compared with baseline and a score $\geq 1$ to the PCSI question: In general, to what degree do you feel "differently" than before the injury (rated from " 0 " [no difference] to " 4 " [major difference])? or asymptomatic if there was no increase in symptoms and a score of zero to the "feeling different" question. ${ }^{4}$

\section{Outcome measures}

CNS vital signs. All participants were administered the CNS Vital Signs computerized cognitive battery. This test provides a rapid assessment of cognitive abilities. It has been normed for use in children and adults between 7 and 90 years and has adequate testretest reliability in children and adolescents (Pearson's $r=0.63$ 0.82 ), and concurrent validity with traditional neuropsychological measures. ${ }^{28,29}$ It is a valid measure of cognitive skills in children with neurological disorders, ${ }^{30}$ including TBI. ${ }^{31,32}$ The Neurocognition Index (NCI) is a summary score that is the average of five domain scores: Composite Memory, Psychomotor Speed, Reaction Time, Complex Attention, and Cognitive Flexibility. All domain scores on the CNS Vital Signs are normalized to a mean of 100 and standard deviation of 15 . The NCI was used to provide an overall estimate of cognitive function. Complex attention, cognitive flexibility and executive function domains are sensitive to change in mTBI. ${ }^{28}$

\section{Image acquisition and pre-processing}

All participants received 3.0T MRI scans on a GE MR750w Discovery scanner using a 32-channel head coil (MRI Instruments). The imaging protocol included a 5-min 30-sec anatomical 3D $\mathrm{T}_{1}$
BRAVO with $0.8 \mathrm{~mm}^{3}$ isotropic resolution over the whole brain. The vendor-supplied 3D pseudo-continuous ASL sequence was performed with post-labeling delay of $2.0 \mathrm{sec}$. In-plane spatial resolution was $3.2 \mathrm{~mm}^{2}$. Thirty-four $3.5-\mathrm{mm}$ thick slices were acquired in a total scan time of $5 \mathrm{~min}$. The 3D ASL scan was automatically processed into quantitative $\mathrm{CBF}$ maps using the scanner-integrated pipeline with default settings (partition coefficient of 0.9 ; blood $\mathrm{T}_{1}$ of $1.6 \mathrm{sec}$ ). Anatomical BRAVO was segmented into five tissue probability maps (gray matter, white matter, cerebral spinal fluid, skull, skin) using Statistical Parametric Mapping (SPM) 12 (www.fil.ion.ucl.ac.uk/ $\mathrm{spm} / \mathrm{software} / \mathrm{spm} 12$ ). The raw perfusion-weighted ASL image and CBF maps were co-registered to the gray matter probability density map to account for motion between the $\mathrm{T}_{1}$ and ASL scans. The CBF maps were then normalized to Montreal Neurological Institute space.

\section{Statistical analysis}

Normality tests (Kolmogorov-Smirnov) for all measures were conducted using IBM SPSS version 22.0. Skewed data (PCSI) were analyzed with non-parametric tests. Between-group effect sizes were calculated using Cohen's $f_{B}^{2}$ effect sizes, and 0.02, 0.15, and 0.35 are termed small, medium, and large, respectively. ${ }^{33}$ Correlations between $\mathrm{CBF}$ and age, group, family income (as a marker of socioeconomic status [SES]), and the NCI were performed. SPM 12 was used to analyze the processed CBF maps. A gray-matter mask was created using a tissue probability map where gray matter probability was $>40 \%$ to constrain the statistical comparisons to gray matter only. A one-way analysis of variance (ANOVA) was used to compare the symptomatic, asymptomatic, and healthy control groups. Post hoc independent samples $t$ tests were subsequently used to detect differences between patient groups. Only regions that were $p<0.05$ and voxels clusters $>100$ were considered. General linear models with main effects for sex, group/mTBI, PCSI score, NCI, and global CBFwere fitted to the data and preinjury PCSI was included as a covariate.

\section{Results}

Eighty-four participants consented to the study; see Figure 1. Ten participants either could not tolerate MRI or ended the MRI session before ASL-MRI was performed (6 symptomatic, 2 asymptomatic, and 2 control participants). One healthy control participant was excluded due to the presence of a benign tumor. Neuroimaging was obtained in 72 participants (mean age: 14.1 years; $95 \%$ confidence intervals (CIs): 13.5-14.8). The sociodemographic information of the participants is shown in Table 1. The groups were similar in age,

Table 1. Demographic Information of Participants Who Were Symptomatic and Asymptomatic after an mTBI and Healthy Controls

\begin{tabular}{|c|c|c|c|c|c|}
\hline & $\begin{array}{c}\text { Symptomatic }(\mathrm{n}=27) \\
(95 \% C I)\end{array}$ & $\begin{array}{c}\text { Asymptomatic }(\mathrm{n}=24) \\
(95 \% \text { CI })\end{array}$ & $\begin{array}{c}\text { Control }(\mathrm{n}=21) \\
(95 \%(C I))\end{array}$ & Statistical test & $\mathrm{P}$ \\
\hline Age (years) & $14.03(12.92-15.13)$ & $14.09(13.10-15.08)$ & $14.40(12.89-15.09)$ & - & - \\
\hline Male $(\%)$ & 44 & 50 & 40 & - & - \\
\hline Right-handed (\%) & 78 & 88 & 95 & $\chi^{2}=2.89$ & 0.236 \\
\hline Mean family income (CA\$) & $117865(108,325-127,405)$ & $125000(100,962-149,037)$ & $123433(96,107-150,759)$ & $\mathrm{F}(2,71)=0.13$ & 0.879 \\
\hline Previous concussion $(n)$ & 12 & 4 & 0 & $\chi^{2}=4.55$ & 0.033 \\
\hline $\begin{array}{l}\text { Previous attention } \\
\text { problems }(n)\end{array}$ & 2 & 1 & 0 & $\chi^{2}=0.23$ & 0.633 \\
\hline Pre-injury PCSI, median & 2.0 (range: 0 & 0 (range & 2.0 (range: $0-21)$ & $\mathrm{K}=11$. & 0.003 \\
\hline Time since injury (days) & $40.63(38.45-42.81)$ & $40.08(36.05-44.11)$ & - & $\mathrm{t}(49)=-0.25$ & 0.801 \\
\hline Post-injury PCSI, median & 36.0 (range: 6-22) & 2.5 (range: $0-26$ ) & - & $\mathrm{U}=607$ & $<0.001$ \\
\hline Neurocognition Index & $94.1(88.4-99.9)$ & $104.1(99.9-108.2)$ & $102.7(97.0-108.3)$ & $F(2,67)=5.75$ & 0.005 \\
\hline
\end{tabular}

The symptomatic group had worse cognitive function post-injury when compared with the asymptomatic and healthy control groups.

CA\$: Canadian dollars; CI, confidence intervals; mTBI, mild traumatic brain injury; PCSI, Post-Concussion Symptom Inventory score. 


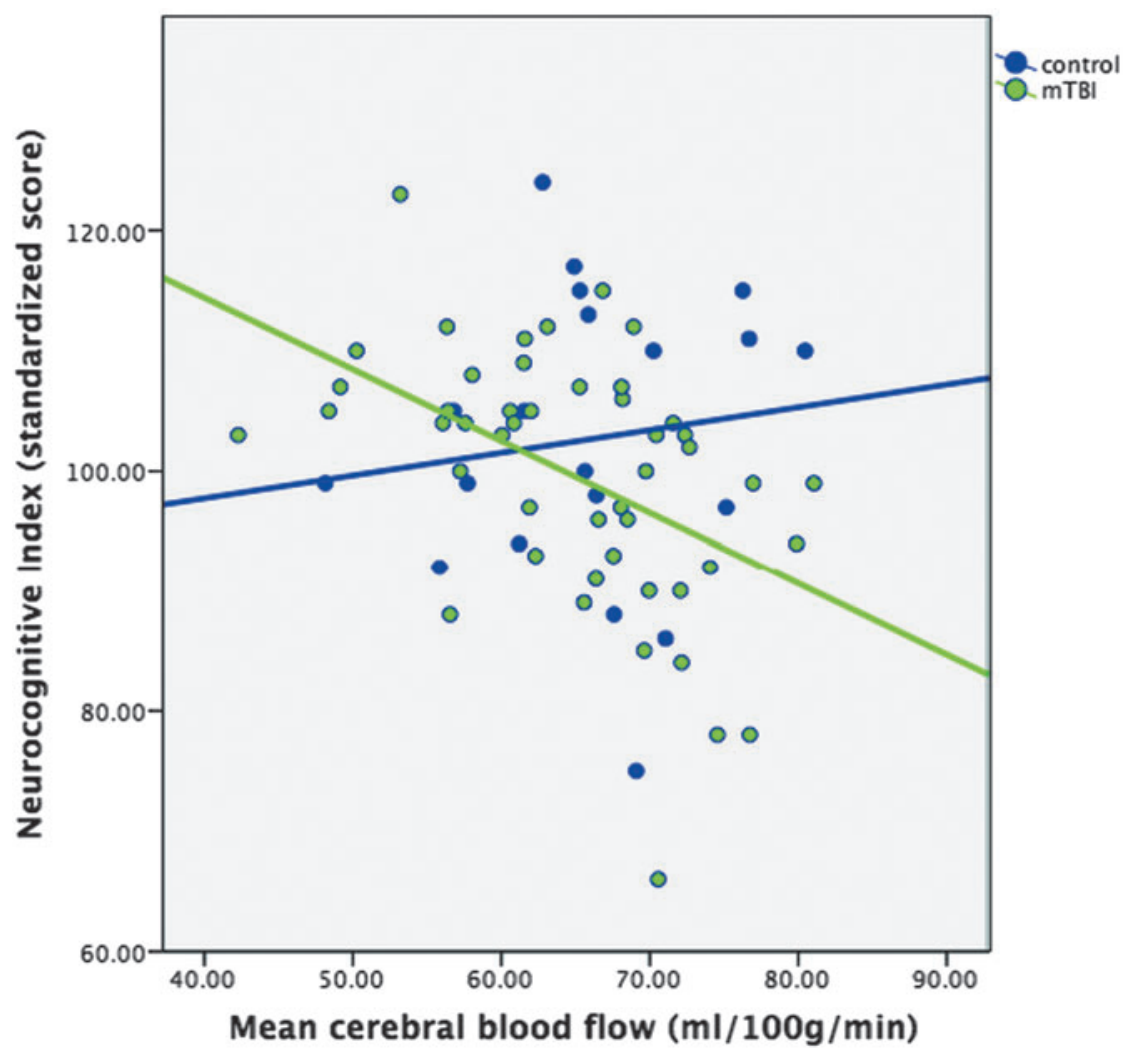

FIG. 2. A scatterplot demonstrating the correlation between cognitive function (Neurocognition Index) and global CBF. Cognitive function was predicted by CBF only in the $\mathrm{mTBI}$ group (significant interaction effect between $\mathrm{CBF}$ and $\mathrm{mTBI} ; \mathrm{beta}=-2.16, p=0.028$ ), $\mathrm{R}^{2}=0.165, p=0.008$. CBF, cerebral blood flow; mTBI, mild traumatic brain injury.

sex, and family income. Although the causes of injury were heterogeneous, mTBI was attributable to a sport-related injury in $60 \%$ of participants (54\% symptomatic vs. $45 \%$ asymptomatic groups). Participants in the symptomatic group were more likely to have a history of previous concussion compared with the asymptomatic $\operatorname{group}\left(\chi^{2}=4.55 ; p=0.033\right)$. The concussions were $>3$ months before the present injury, and all participants reported recovery from any previous concussion. Symptomatic participants were not more likely to have a history of attentional problems compared with the asymptomatic group. In keeping with their symptomatic status, postinjury PSCI scores were higher in the symptomatic group compared with the asymptomatic group. The scores on the NCI differed between groups $(\mathrm{F}(2,67), 5.75 ; p=0.005)$, and were lower in the symptomatic group $($ mean $=94.2,95 \%$ CIs: 88.4-99.9; Table 1$)$.

All participants were normotensive on the day of testing. Imaging was performed at the same time post-injury in symptomatic and asymptomatic participants: 40 (standard deviation [SD] 7.6) days $(p=0.801)$. Significant differences in CBF were found between all groups at 40 days post-injury. Mean global CBF differed significantly between groups: the symptomatic group had a higher global CBF, 70.23(SD 6.80) $\mathrm{mL} / 100 \mathrm{~g} / \mathrm{min}$, compared with the healthy control group, 65.94 (SD 7.94) $\mathrm{mL} / 100 \mathrm{~g} / \mathrm{min}$, and the asymptomatic group had a lower global CBF, 59.57 (SD 7.13) $\mathrm{mL} / 100 \mathrm{~g} /$ $\min (\mathrm{F}(2,71) 9.734 p<0.001)$. The differences of CBF between

Table 2. Regression Model Summary Predicting Post-Injury Post-Concussion Symptom Inventory Score

\begin{tabular}{|c|c|c|c|c|c|c|}
\hline$R$ & & $R$ square & Adjusted R square & $d f 1$ & $d f 2$ & Sig. F change \\
\hline \multicolumn{2}{|c|}{.0676} & 0.457 & 0.424 & 4 & 64 & $<0.001$ \\
\hline & & Standardized coefficients & & & & CIs \\
\hline \multicolumn{2}{|c|}{ Model } & Beta & $\mathrm{t}$ & Sig. & Lower bound & Upper bound \\
\hline \multirow[t]{5}{*}{1} & (Constant) & & 0.960 & 0.341 & -80.592 & 229.801 \\
\hline & Mean CBF & -0.487 & -1.237 & 0.221 & -3.775 & 0.888 \\
\hline & TBI & -1.258 & -1.660 & 0.102 & -156.825 & 14.482 \\
\hline & Pre-injury PCSI & 0.372 & 3.934 & $<0.001$ & 0.580 & 1.776 \\
\hline & $\mathrm{TBI} \times \mathrm{CBF}$ & 1.765 & 2.119 & 0.038 & 0.078 & 2.661 \\
\hline
\end{tabular}

CBF, cerebral blood flow; CIs, confidence intervals; PCSI, Post-Concussion Symptom Inventory score; TBI, traumatic brain injury. 
Table 3. Regression Model Summary Predicting Neurocognition Index

\begin{tabular}{|c|c|c|c|c|c|c|}
\hline$R$ & & $R$ square & Adjusted R square & $d f 1$ & $d f 2$ & Sig. F change \\
\hline \multicolumn{2}{|c|}{0.442} & 0.196 & 0.158 & 3 & 64 & 0.003 \\
\hline & & Standardized coefficients & & & & CIs \\
\hline \multicolumn{2}{|c|}{ Model } & Beta & $\mathrm{t}$ & Sig. & Lower bound & Upper bound \\
\hline \multirow[t]{4}{*}{1} & (Constant) & & 1.945 & 0.056 & -1.636 & 122.131 \\
\hline & Mean CBF & 0.511 & 1.483 & 0.143 & -0.238 & 1.609 \\
\hline & Group & 2.263 & 2.057 & 0.044 & 0.891 & 60.660 \\
\hline & Group $\times \mathrm{CBF}$ & -2.767 & -2.281 & 0.026 & -0.937 & -0.062 \\
\hline
\end{tabular}

Group-coded Control (1), Asymptomatic (2), and Symptomatic (3).

$\mathrm{CBF}$, cerebral blood flow; CIs, confidence intervals.

groups had a large effect size (Cohen's $f^{2}=0.422$ ). Within the mTBI groups, the number of previous concussions was not significantly correlated with global CBF (Pearson's $r=-.165 ; p=0.350$ ).

CBF correlated with the sex (female) $(\rho 0.284 ; p=0.017)$, PSCI $(\rho 0.290 ; p=0.016)$, Group $(\rho 0.278 ; p=0.021)$, and NCI $(r-0.371$; $p=0.002)$ but not with age or SES. Variables that correlated at the $p<0.1$ significance level in univariate analyses (Sex, Group, PCSI, and $\mathrm{CBF}$ ) were included in a multiple linear regression analysis to predict PCSI and NCI. There were significant interaction effects between group $\times \mathrm{CBF}$, and TBI $\times \mathrm{CBF}$ (see Fig. 2). Pre-injury PCSI (beta $=0.372, p<0.001)$ and $\mathrm{mTBI} \times \mathrm{CBF}($ beta $=1.765, p=0.038)$ were significant predictors of post-injury PCSI, but not CBF or TBI independently. Overall, the model predicted $42 \%$ of the variance in post-injury post-concussion symptoms (adjusted $\mathrm{R}^{2}=0.424$; $p<0.001$ ), see Table 2. NCI could be predicted by Group (control, asymptomatic, symptomatic; beta $=2.336, p=0.0243$ ) and Group $\times$ CBF (beta $=-2.134, p=0.037$ ), with the overall model predicting $16 \%$ of the variance (adjusted $\mathrm{R}^{2}=0.158 ; p=0.003$ ), see Table 3 .

Regional CBF analyses demonstrated that those children who were symptomatic at 5 to 6 weeks post-injury had regions of higher $\mathrm{CBF}$ when compared with controls, especially in the bilateral inferior frontal and occipital regions (overall $\mathrm{p}_{\text {crit }}<0.05$, voxel cluster-level $>100$, see Fig. 3). There were no regions of decreased perfusion when compared with asymptomatic participants or healthy controls (Fig. 4). In contrast, asymptomatic participants had lower $\mathrm{CBF}$ especially in the inferior temporal and parietal regions compared with healthy controls (overall $\mathrm{p}_{\text {crit }}<0.05$, voxel clusterlevel $>100$, see Fig. 5 at 5 to 6 weeks post-injury). There were no significant voxel clusters where asymptomatic patients had greater perfusion than healthy controls.

\section{Discussion}

We have demonstrated significant differences in cerebral perfusion following mTBI that is dependent on recovery. Our results indicate that cerebral perfusion is significantly higher in children with mTBI who are symptomatic when compared with healthy controls. Further, we have also demonstrated that children who have clinically "recovered" may still have ongoing changes in cerebral perfusion, that is, decreased cerebral perfusion, and so may not be fully "neurologically" recovered.

$\mathrm{CBF}$ is related to cerebral perfusion pressure, blood viscosity, vessel length, and inversely related to the radius of the blood vessel (Hagen-Poiseuielle's law). ${ }^{34}$ In practice, especially in $\mathrm{mTBI}$, changes in $\mathrm{CBF}$ are related to changes in cerebral perfusion pressure and vessel reactivity, that is, cerebral autoregulation, as blood viscosity and vessel length are unchanged. CBF alterations are well described following moderate and severe TBI, and occur more commonly in children than adults. ${ }^{12,35-37}$ Following severe forms of TBI, there is an acute decrease in $\mathrm{CBF}$ in the first day (the

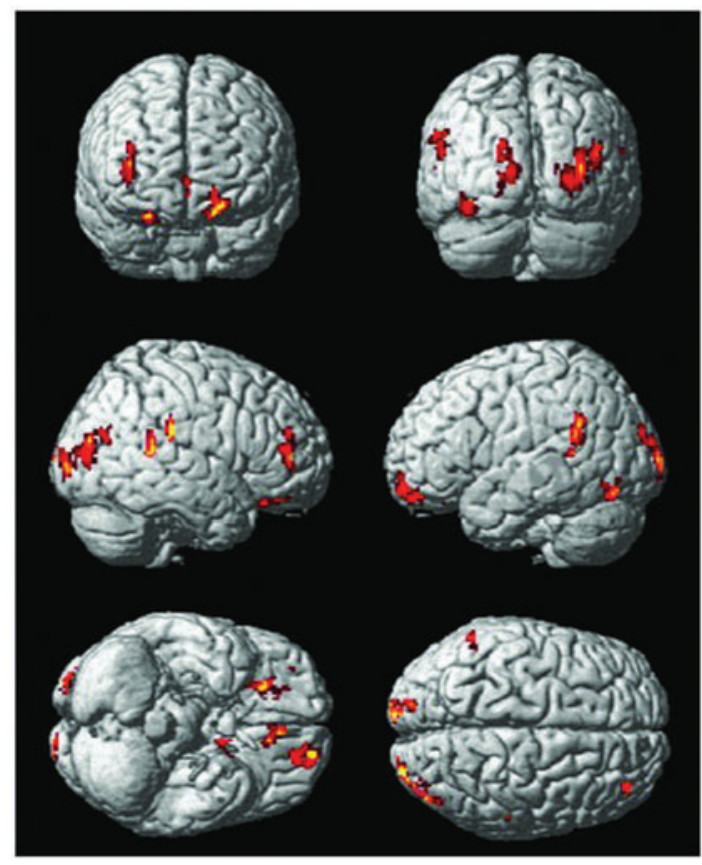

\begin{tabular}{|l|c|c|}
\hline MNI Coordinates (Anatomical area) & Cluster Size (Voxels) & p-value \\
\hline $54-3024$ (R supramar ginal) & 103 & 0.028 \\
\hline$-52-5022$ (L middle temporal) & 144 & 0.011 \\
\hline 364212 (R middle frontal) & 108 & 0.025 \\
\hline $56-4212$ (R middle temporal) & 117 & 0.020 \\
\hline $34-8210$ (R middle occipital) & 299 & 0.001 \\
\hline $2628-22$ (R infer ior frontal, orbital) & 105 & 0.027 \\
\hline$-8-1028$ (L superior occipital) & 141 & 0.012 \\
\hline$-1850-18$ (L supraorbital frontal) & 151 & 0.010 \\
\hline$-832-14$ (L rectus, inferior frontal) & 141 & 0.012 \\
\hline$-34-74$-14 (L fusiform) & 132 & 0.015 \\
\hline
\end{tabular}

FIG. 3. The regions where cerebral perfusion is significantly greater in children who remained symptomatic at 40 days following an mTBI when compared with healthy controls are shown. The cluster size and coordinates of these areas are reported in the table. MNI, Montreal Neurological Institute; mTBI, mild traumatic brain injury. 


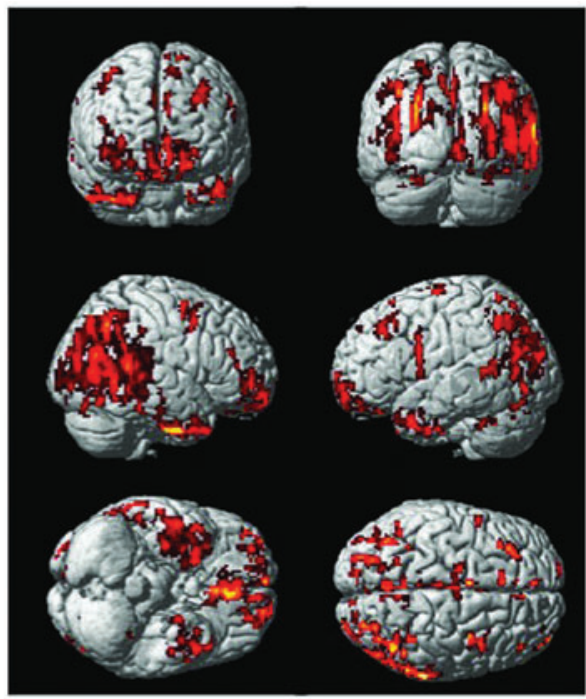

\begin{tabular}{|c|c|c|}
\hline MNI Coordinates (mm) & Cluster Size (Voxels) & p-value \\
\hline$-438-12 \mathrm{~L}$ medial orbital, frontal & 1802 & 0.000 \\
\hline $36060 \mathrm{R}$ middle frontal & 206 & 0.003 \\
\hline$-283438 \mathrm{~L}$ middle frontal & 213 & 0.003 \\
\hline$-103630 \mathrm{~L}$ medial superior frontal & 262 & 0.001 \\
\hline$-56418 \mathrm{~L}$ precentral & 190 & 0.004 \\
\hline $66-3812 \mathrm{R}$ superior temporal & 3155 & 0.000 \\
\hline $52-8-38 \mathrm{R}$ inferior temporal & 845 & 0.000 \\
\hline$-48-488 \mathrm{~L}$ middle temporal & 166 & 0.007 \\
\hline$-48-10-38 \mathrm{~L}$ inferior temporal & 385 & 0.000 \\
\hline $63628 \mathrm{R}$ anterior cingulus & 127 & 0.015 \\
\hline$-62422 \mathrm{~L}$ anterior cingulus & 109 & 0.023 \\
\hline$-24-7238$ L superior occipital & 562 & 0.000 \\
\hline$-12-4254 \mathrm{~L}$ precuneus & 613 & 0.000 \\
\hline$-4-6632 \mathrm{~L}$ precuneus & 1942 & 0.000 \\
\hline$-36-6040 \mathrm{~L}$ angular & 724 & 0.000 \\
\hline$-30-60-10 \mathrm{~L}$ fusiform & 454 & 0.000 \\
\hline
\end{tabular}

FIG. 4. The regions where perfusion in the symptomatic group is significantly greater than the asymptomatic group at 40 days post-injury are shown. The cluster size and coordinates of these areas are given in the table. MNI, Montreal Neurological Institute.

hypoperfusion phase), followed by a period of increased CBF (the hyperemia phase lasting 1 to 3 days), and subsequently followed by another decrease in CBF that lasts from a few days to a few weeks. ${ }^{37,38}$ In mTBI, however, much less is known about CBF changes, and its relationship to cerebral metabolic rate, vascular reactivity, and outcome. ${ }^{12}$

We report the largest study to date examining cerebral perfusion using pseudo-continuous ASL in children at precise times after mTBI while controlling for the effects of age. We found that children differed significantly in their pattern of cerebral perfusion depending on their recovery trajectories. This difference in cerebral perfusion was greatest between the symptomatic and asymptomatic groups. Indeed, post-injury PCSI scores at one month were significantly related to $\mathrm{CBF}$ in the presence of mTBI, and pre-injury PCSI scores. Those who remained symptomatic at one-month post-injury had higher $\mathrm{CBF}$ when compared with those who had recovered and healthy controls. Although higher CBF has been reported before in mTBI, it has been in the setting of acute mTBI. ${ }^{39}$ There are actually few studies examining CBF in the time period when symptoms begin to resolve in most children, that is, 4 to 6 weeks post-injury. ${ }^{4}$ A strength of our study is that

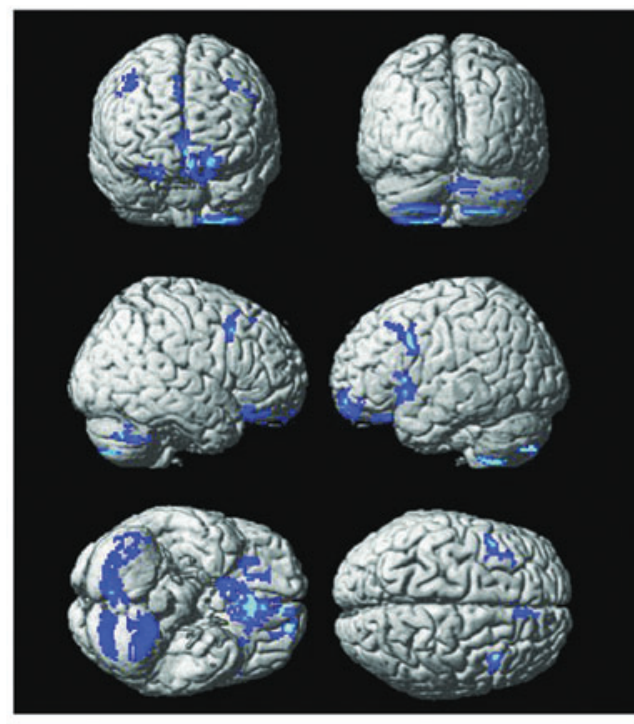

\begin{tabular}{|l|c|c|}
\hline \multicolumn{1}{|c|}{ MNI Coordinates (mm) } & Cluster Size (Voxels) & p-value \\
\hline$-1858-12$ (L supraorbital, frontal) & 126 & 0.011 \\
\hline$-216-20$ ( L rectus, frontal) & 1483 & 0.000 \\
\hline$-3016-12$ (L insula) & 295 & 0.000 \\
\hline 361438 (R middle frontal) & 130 & 0.010 \\
\hline-441432 (L pre-central) & 209 & 0.002 \\
\hline $3228-16$ (R inferior frontal, orbital) & 108 & 0.017 \\
\hline$-20-40-54$ (L cerebellum) & 209 & 0.002 \\
\hline $10-72-48$ (R cerebellum) & 129 & 0.010 \\
\hline $40-62-30$ (R crus cerebellum) & 126 & 0.011 \\
\hline
\end{tabular}

FIG. 5. The regions where cerebral perfusion is significantly decreased in children who were asymptomatic at 40 days following an mTBI when compared with healthy controls are shown. The cluster size and coordinates of the areas are reported in the table (overall pcrit $<0.05$, voxel cluster-level $>100$ ). MNI, Montreal Neurological Institute; mTBI, mild traumatic brain injury.

imaging was performed at the same time post-injury (40 days) in all participants.

Asymptomatic children, in contrast, had lower cerebral perfusion when compared with healthy controls. This finding of lower cerebral perfusion is similar to the finding reported by Maugans and colleagues, who investigated global CBF in 11 children with a sports-related concussion at one-month post-injury. ${ }^{16}$ Ten of the 11 children studied reported resolution of their symptoms and their overall global CBF was lower compared with controls (mean $=39.2$ vs. $48.0 \mathrm{~mL} / 100 \mathrm{~g}$ per min). Recovery status in the study by Maugans and associates and in our study was based on symptom report alone and is consistent with clinical practice. Symptoms had to have returned to pre-injury levels (or below) to be considered asymptomatic. This relative decrease in $\mathrm{CBF}$ in the recovery stages of mTBI is concerning as most children are returning to normal activities by this stage. Exactly what these changes mean in the long term and their impact on return to normal activities requires further exploration.

Our findings are not consistent with those reported by Wang and colleagues, who found that adolescents with PCS had lower CBF compared with controls, especially in the frontotemporal regions. ${ }^{13}$ Wang and co-workers, however, studied symptomatic adolescents at a later time-point (mean: 7.8 months) following mTBI. It is very possible that perfusion could continue to decrease over time in the symptomatic children. Such a decrease in CBF has been 
demonstrated in a longitudinal study of collegiate athletes and may relate to psychological symptoms over time. ${ }^{15}$ This is worthy of future longitudinal study in children to determine if such changes are related to symptom evolution.

Cerebral perfusion in the setting of an mTBI at 4 to 6 weeks was the best predictor of cognition function-lower cognitive function was associated with higher CBF. Decreases in temporal and parietal cortical perfusion have previously been associated with a poorer cognitive outcome in acute $\mathrm{mTBI}^{40}$ but in the subacute stages. Chronically, cognitive function has also been associated with decreased perfusion in the thalami and basal ganglia ${ }^{41}$ and could explain some of the persistent changes in attention processing following mTBI. Lower cognitive function was associated with higher CBF in mTBI participants only, and although this could have been present pre-injury, it could alternatively be indicative of a relative $\mathrm{CBF}-$ metabolic mismatch.

The neurobiology of changes in CBF following mTBI are unknown but are substantiated by other markers of autonomic dysfunction and vascular reactivity (i.e., heart rate variability, orthostatic hypotension), which have been reported both acute$1 y^{12,42}$ and in chronic PCS. ${ }^{43,44}$ In moderate/severe TBI decreases in cerebral perfusion correlate with tissue loss. ${ }^{45}$ However, mTBI is not associated with such tissue loss. The scattered areas of perfusion changes observed in our study suggest that CBF changes are not predominantly due to abnormal cerebral autoregulation. However, as CBF is closely linked to neuronal metabolism (CBFmetabolic coupling) and occurs mainly at the microvascular level, the changes observed following mTBI could be due to a relative uncoupling of neuronal activity to the astrocytic and vascular components of the neurovascular unit. ${ }^{46}$ The paradoxical finding of increased CBF in mTBI participants with lower NCI could be suggestive of lower cognitive functioning in those with greater metabolic uncoupling. CBF alterations could also be due to traumatic microvascular injury. ${ }^{47}$ How $\mathrm{CBF}$ alterations relate to microstructural, network, or metabolic disturbances and their relationship to clinical symptoms in children with mTBI requires further study. ${ }^{14,48-51}$

\section{Limitations}

There are several limitations to this study. It is possible that our sample does not reflect the true population of mTBI due to possible recruitment and selection biases. Alterations in cerebral perfusion are not specific to TBI and can be found in individuals with disorders such as attention deficit and hyperactivity disorder and mood disorders. ${ }^{52,53}$ This is relevant to our study as children with TBI are more likely to have pre-existing attention deficits and we did not exclude these children from our study. Further, we did not use a non-TBI injured control group, which may have mitigated some of the effects of not controlling for attention problems. ${ }^{54,55}$ It is also possible that the differences in cerebral perfusion observed are related to cognitive function and independent of the injury. Similarly, this study reports differences in $\mathrm{CBF}$ at a single time-point post-injury. It is possible that these difference were present preinjury, although it is difficult to explain why children with lower $\mathrm{CBF}$ are more likely to have a good outcome following mTBI. Longitudinal studies tracking CBF changes from pre-injury to 6 months post-injury would be ideal to investigate this further. Despite these limitations, this study is generalizable to the population of children with PCS seen in the clinician's office at 1 to 2 months post-injury who often also have comorbid pre-existing mild learning difficulties.

\section{Conclusion}

In summary, recovery from mTBI is likely to be a dynamic process and may differ in children when compared with adults. Altered patterns of cerebral perfusion are seen in children with mTBI and are associated with their recovery trajectory. Children who are still symptomatic at 5 to 6 weeks post-injury displayed higher cerebral perfusion. Children who quickly "recovered" after their mTBI, however, show lower cerebral perfusion relative to healthy controls, especially in the temporal lobes, perhaps suggesting that cerebral recovery is still ongoing. Longitudinal investigations of vascular reactivity and cerebral metabolism are needed to identify the underlying pathogenesis of cerebral perfusion changes over time and their association with outcomes in children with mTBI.

\section{Acknowledgments}

We would like to acknowledge the research coordinator, Ms. Brenda Turley, for her invaluable help and endless energy and without whom this work would not be possible. We would also like to acknowledge the research students and assistants: Trevor Seeger, Erica Crowe, Trevor Low, Tina Samuel, Mai Vo, and Aneesh Khetani.

Dr. Barlow conceptualized and designed the study, supervised data collection, performed secondary analyses, drafted the initial manuscript, and approved the final manuscript as submitted. Mr. Marcil carried out the initial analyses, reviewed and revised the manuscript, and approved the final manuscript as submitted. Dr. Dewey helped to design the study, reviewed and revised the manuscript, and approved the final manuscript as submitted. Dr. Carlson carried out the initial analyses, reviewed and revised the manuscript, and approved the final manuscript as submitted. Drs. MacMaster and Brooks coordinated and supervised data collection, reviewed and revised the manuscript, and approved the final manuscript as submitted. Dr. Lebel carried out the initial analyses, critically reviewed and revised the manuscript, and approved the final manuscript as submitted.

\section{Author Disclosure Statement}

Drs. Barlow, Dewey, MacMaster, and Mr. Marcil have no competing financial interests. Dr. Brooks receives royalties for the sales of a pediatric forensic textbook (Oxford University Press) and pediatric neuropsychological tests (PAR Inc.). He has previously received support (in-kind test credits) from CNS Vital Signs. Dr. Lebel is a stakeholder in GE Healthcare. This study was funded by the Canadian Institutes of Health Research (grant number: 293375); the Faculty of Medicine, University of Calgary; and the Markin Undergraduate Student Research Program, University of Calgary. These funding sources had no role in the design of this study and did not have any role during its execution, analyses, interpretation of the data, or decision to submit results. The design, management, analysis, and reporting of the study were entirely independent of any manufacturers of melatonin.

\section{References}

1. McKinlay, A., Grace, R.C., Horwood, L.J., Fergusson, D.M., Ridder, E.M., and MacFarlane, M.R. (2008). Prevalence of traumatic brain injury among children, adolescents and young adults: prospective evidence from a birth cohort. Brain Inj. 22, 175-181.

2. Ryu, W.H.A., Feinstein, A., Colantonio, A., Streiner, D.L., and Dawson, D.R. (2009). Early identification and incidence of mild TBI in Ontario. Can. J. Neurol. Sci. 36, 429-435. 
3. Barlow, K.M., Crawford, S., Stevenson, A., Sandhu, S.S., Belanger, F., and Dewey, D. (2010). Epidemiology of postconcussion syndrome in pediatric mild traumatic brain injury. Pediatrics 126, e374-e381.

4. Barlow, K.M., Crawford, S., Brooks, B.L., Turley, B., and Mikrogianakis, A. (2015). The incidence of postconcussion syndrome remains stable following mild traumatic brain injury in children. Pediatr. Neurol. 53, 491-497.

5. Barkhoudarian, G., Hovda, D.A., and Giza, C.C. (2011). The molecular pathophysiology of concussive brain injury. Clin. Sports Med. 30, 33-48, vii-iii.

6. Yeates, K.O., Taylor, H.G., Rusin, J., Bangert, B., Dietrich, A., Nuss, K., and Wright, M. (2012). Premorbid child and family functioning as predictors of post-concussive symptoms in children with mild traumatic brain injuries. Int. J. Dev. Neurosci. 30, 231-237.

7. Kozlowski, K.F., Graham, J., Leddy, J.J., Devinney-Boymel, L., and Willer, B.S. (2013). Exercise intolerance in individuals with postconcussion syndrome. J. Athl. Train. 48, 627-635.

8. Barlow, K.M. (2016). Postconcussion syndrome: a review. J. Child Neurol. 31, 57-67.

9. Keightley, M.L., Singh Saluja, R., Chen, J.K., Gagnon, I., Leonard, G., Petrides, M., and Ptito, A. (2014). A functional magnetic resonance imaging study of working memory in youth after sports-related concussion: is it still working? J. Neurotrauma 31, 437-451.

10. Slobounov, S.M., Gay, M., Zhang, K., Johnson, B., Pennell, D., Sebastianelli, W., Horovitz, S., and Hallett, M. (2011). Alteration of brain functional network at rest and in response to YMCA physical stress test in concussed athletes: RsFMRI study. Neuroimage 55 , 1716-1727.

11. Len, T.K., Neary, J.P., Asmundson, G.J.G., Candow, D.G., Goodman, D.G., Bjornson, B., and Bhambhani, Y.N. (2013). Serial monitoring of $\mathrm{CO} 2$ reactivity following sport concussion using hypocapnia and hypercapnia. Brain Inj. 27, 346-353.

12. Len, T.K., and Neary, J.P. (2011). Cerebrovascular pathophysiology following mild traumatic brain injury. Clin. Physiol. Funct. Imaging 31, 85-93.

13. Wang, Y., West, J.D., Bailey, J.N., Westfall, D.R., Xiao, H., Arnold, T.W., Kersey, P.A., Saykin, A.J., and McDonald, B.C. (2015). Decreased cerebral blood flow in chronic pediatric mild TBI: an MRI perfusion study. Dev. Neuropsychol. 40, 40-44.

14. Churchill, N., Hutchison, M., Richards, D., Leung, G., Graham, S., and Schweizer, T.A. (2016). Brain structure and function associated with a history of sport concussion: a multi-modal MRI study. J Neurotrauma. [Epub ahead of print.]

15. Meier, T.B., Bellgowan, P.S., Singh, R., Kuplicki, R., Polanski, D.W., and Mayer, A.R. (2015). Recovery of cerebral blood flow following sports-related concussion. JAMA Neurol. 72, 530-538.

16. Maugans, T.A., Farley, C., Altaye, M., Leach, J., and Cecil, K.M. (2012). Pediatric sports-related concussion produces cerebral blood flow alterations. Pediatrics 129, 28-37.

17. Petcharunpaisan, S., Ramalho, J., and Castillo, M. (2010). Arterial spin labeling in neuroimaging. World J. Radiol. 2, 384-398.

18. Wang, J., and Licht, D.J. (2006). Pediatric perfusion MR imaging using arterial spin labeling. Neuroimaging Clin. N. Am. 16, 149-167, ix.

19. Wang, Y., Saykin, A.J., Pfeuffer, J., Lin, C., Mosier, K.M., Shen, L., Kim, S., and Hutchins, G.D. (2011). Regional reproducibility of pulsed arterial spin labeling perfusion imaging at 3T. Neuroimage 54, 1188-1195.

20. Barlow, K.M., Dewey, D., Urban, K.J., Seeger, T., Kirton, A., MacMaster, F., Brooks, B., Mikrogianakis, A., Crawford, S., NetelAguirre, A., Johnson, D., Kirk, V., Zemek, R., and Buchhalter, J. (2014). PlayGame: a randomized, double blind, placebo contolled trial of Melatonin for the treatment of post concussion syndrome in youth. J. Neurotrauma 31, A59-A60.

21. Moran, L.M., Taylor, H.G., Rusin, J., Bangert, B., Dietrich, A., Nuss, K.E., Wright, M., Minich, N., and Yeates, K.O. (2012). Quality of life in pediatric mild traumatic brain injury and its relationship to postconcussive symptoms. J. Pediatr. psychol. 37, 736-744.

22. Taylor, H.G., Dietrich, A., Nuss, K., Wright, M., Rusin, J., Bangert, B., Minich, N., and Yeates, K.O. (2010). Post-concussive symptoms in children with mild traumatic brain injury. Neuropsychology 24, 148-159.

23. Bodin, D., Yeates, K.O., and Klamar, K. (2012). Definition and classification of concussion, in: Pediatric and Adolescent Concussion:
Diagnosis, Management, and Outcome. J.N. Apps J N and K.D. Walter (eds). Springer: New York, pps. 9-20.

24. Wang, J., Licht, D.J., Jahng, G.H., Liu, C.S., Rubin, J.T., Haselgrove, J., Zimmerman, R.A., and Detre, J.A. (2003). Pediatric perfusion imaging using pulsed arterial spin labeling. J. Magn. Reson. Imaging $18,404-413$.

25. Barlow, K.M. (2016). Post-concussion syndrome: A review. J. Child Neurol. 31, 57-67.

26. Sady, M.D., Vaughan, C.G., and Gioia, G.A. (2014). Psychometric characteristics of the postconcussion symptom inventory in children and adolescents. Arch. Clin. Neuropsychol. 29, 348-363.

27. Gioia, G.A., Janusz, J.A., Isquith, P.K., and Vincent, D. (2008). Psychometric properties of the parent and teacher post-concussion symptom inventory (PCSI) for children and adolescents. J. Int. Neuropsychol. Soc. 14, 204.

28. Gualtieri, C.T., and Johnson, L.G. (2006). Reliability and validity of a computerized neurocognitive test battery, CNS Vital Signs. Arch. Clin. Neuropsychol. 21, 623-643.

29. Brooks, B.L., and Sherman, E.M. (2012). Computerized neuropsychological testing to rapidly evaluate cognition in pediatric patients with neurologic disorders. J. Child Neurol. 27, 982-991.

30. Brooks, B.L., and Barlow, K.M. (2011). A methodology for assessing treatment response in Hashimoto's encephalopathy: a case study demonstrating repeated computerized neuropsychological testing. J. Child Neurol. 26, 786-791.

31. Brooks, B.L., Khan, S., Daya, H., Mikrogianakis, A., and Barlow, K.M. (2014). Neurocognition in the emergency department after a mild traumatic brain injury in youth. J. Neurotrauma 31, 1744-1749.

32. Brooks, B.L., and Barlow, K.M. (2011). A methodology for assessing treatment response in Hashimoto's encephalopathy: a case study demonstrating repeated computerized neuropsychological testing. J. Child Neurol. 26, 786-791.

33. Cohen, J. (1988). Statistical Power Analysis for the Behavioral Sciences, 2nd ed. Lawrence Erlbaum Associates.

34. Ku, D.N. and Zhu, C. (1993). Hemodynamic Forces and Vascular Cell Biology. CRC Press, RG Landes Co.: Austin, TX.

35. DeWitt, D.S., and Prough, D.S. (2003). Traumatic cerebral vascular injury: the effects of concussive brain injury on the cerebral vasculature. J. Neurotrauma 20, 795-825.

36. Lang, E.W., Lagopoulos, J., Griffith, J., Yip, K., Yam, A., Mudaliar, Y., Mehdorn, H.M., and Dorsch, N.W. (2003). Cerebral vasomotor reactivity testing in head injury: the link between pressure and flow. J. Neurol. Neurosurg. Psychiatry 74, 1053-1059.

37. Adelson, P.D., Srinivas, R., Chang, Y., Bell, M., and Kochanek, P.M. (2011). Cerebrovascular response in children following severe traumatic brain injury. Childs Nerv. Syst. 27, 1465-1476.

38. Martin, N.A., Patwardhan, R.V., Alexander, M.J., Africk, C.Z., Lee, J.H., Shalmon, E., Hovda, D.A., and Becker, D.P. (1997). Characterization of cerebral hemodynamic phases following severe head trauma: hypoperfusion, hyperemia, and vasospasm. J. Neurosurg. 87, 9-19.

39. Doshi, H., Wiseman, N., Liu, J., Wang, W., Welch, R.D., O’Neil, B.J., Zuk, C., Wang, X., Mika, V., Szaflarski, J.P., Haacke, E.M., and Kou, Z. (2015). Cerebral hemodynamic changes of mild traumatic brain injury at the acute stage. PLoS One 10, e0118061.

40. Metting, Z., Spikman, J.M., Rodiger, L.A., and van der Naalt, J. (2014). Cerebral perfusion and neuropsychological follow up in mild traumatic brain injury: acute versus chronic disturbances? Brain Cogn. 86, 24-31.

41. Ge, Y., Patel, M.B., Chen, Q., Grossman, E.J., Zhang, K., Miles, L., Babb, J.S., Reaume, J., and Grossman, R.I. (2009). Assessment of thalamic perfusion in patients with mild traumatic brain injury by true FISP arterial spin labelling MR imaging at 3T. Brain Inj. 23, 666-674.

42. Gall, B., Parkhouse, W., and Goodman, D. (2004). Heart rate variability of recently concussed athletes at rest and exercise. Med. Sci. Sports Exerc. 36, 1269-1274.

43. Abaji, J.P., Curnier, D., Moore, R.D., and Ellemberg, D. (2015). Persisting effects of concussion on heart rate variability during physical exertion. J. Neurotrauma 33, 811-817.

44. Heyer, G.L., Fischer, A., Wilson, J., MacDonald, J., Cribbs, S., Ravindran, R., Pommering, T.L., and Cuff, S. (2016). Orthostatic intolerance and autonomic dysfunction in youth with persistent postconcussion symptoms: a head-upright tilt table study. Clin. J. Sport Med. 26, 40-45. 
45. Kim, J., Whyte, J., Patel, S., Avants, B., Europa, E., Wang, J., Slattery, J., Gee, J.C., Coslett, H.B., and Detre, J.A. (2010). Resting cerebral blood flow alterations in chronic traumatic brain injury: an arterial spin labeling perfusion FMRI study. J. Neurotrauma 27, 1399-1411.

46. Venkat, P., Chopp, M., and Chen, J. (2016). New insights into coupling and uncoupling of cerebral blood flow and metabolism in the brain. Croat. Med. J57, 223-228.

47. Kenney, K., Amyot, F., Haber, M., Pronger, A., Bogoslovsky, T., Moore, C., and Diaz-Arrastia, R. (2016). Cerebral vascular injury in traumatic brain injury. Exp. Neurol. 275, Pt 3, 353-366.

48. Urban, K.J., Barlow, K.M., Jimenez, J.J., Goodyear, B.G., and Dunn, J.F. (2014). Pediatric post-concussion symptoms are associated with reduced cortical communication as detected with near-infrared spectroscopy. J. Neurotrauma 31, A64-A64.

49. Chamard, E., Théoret, H., Skopelja, E.N., Forwell, L.A., Johnson, A.M., and Echlin, P.S. (2012). A prospective study of physicianobserved concussion during a varsity university hockey season: metabolic changes in ice hockey players. Part 4 of 4 . Neurosurg. Focus 33, E4: 1-7.

50. Chen, J.K., Johnston, K.M., Petrides, M., and Ptito, A. (2008). Neural substrates of symptoms of depression following concussion in male athletes with persisting postconcussion symptoms. Arch. Gen. Psychiatry $65,81-89$.

51. Vagnozzi, R., Signoretti, S., Cristofori, L., Alessandrini, F., Floris, R., Isgro, E., Ria, A., Marziale, S., Zoccatelli, G., Tavazzi, B., Del Bolgia, F., Sorge, R., Broglio, S.P., McIntosh, T.K., and Lazzarino, G. (2010). Assessment of metabolic brain damage and recovery following mild traumatic brain injury: a multicentre, proton magnetic resonance spectroscopic study in concussed patients. Brain 133, 3232-3242.
52. Kim, B.N., Kim, J.W., Kang, H., Cho, S.C., Shin, M.S., Yoo, H.J., Hong, S.B., and Lee, D.S. (2010). Regional differences in cerebral perfusion associated with the alpha-2A-adrenergic receptor genotypes in attention deficit hyperactivity disorder. J. Psychiatry Neurosci. 35, 330-336.

53. Lorberboym, M., Watemberg, N., Nissenkorn, A., Nir, B., and Lerman-Sagie, T. (2004). Technetium 99m ethylcysteinate dimer single-photon emission computed tomography (SPECT) during intellectual stress test in children and adolescents with pure versus comorbid attention-deficit hyperactivity disorder (ADHD). J. Child Neurol. 19, 91-96.

54. Basson, M.D., Guinn, J.E., McElligott, J., Vitale, R., Brown, W., and Fielding, L.P. (1991). Behavioral disturbances in children after trauma. J. Trauma 31, 1363-1368.

55. Max, J.E., Lansing, A.E., Koele, S.L., Castillo, C.S., Bokura, H., Schachar, R., Collings, N., and Williams, K.E. (2004). Attention deficit hyperactivity disorder in children and adolescents following traumatic brain injury. Dev. Neuropsychol. 25, 159-177.

Address correspondence to:

Karen M. Barlow, MSc, MB ChB, MRCPCH

University of Calgary

Section of Neurology, Alberta Children's Hospital 2888 Shaganappi Trail NW

Calgary, Alberta, Canada T3B 6 A8

E-mail: Karen.Barlow@albertahealthservices.ca 